\title{
Stents Farmacológicos para o Tratamento de Coronárias de Fino Calibre: Experiência Muito Tardia (Até 7 Anos) do Registro DESIRE
}

\author{
Ederlon Ferreira Nogueira ${ }^{1}$, Amanda G. M. R. Sousa ${ }^{1}$, J. Ribamar Costa Jr. ${ }^{1}$, Adriana Moreira ${ }^{1}$, \\ Ricardo Costa', Galo Maldonado', Cantídio Campos ${ }^{1}$, Manuel Cano'1, J. Eduardo Sousa'
}

\section{RESUMO}

Introdução: Neste estudo, buscamos avaliar os resultados clínicos de pacientes com lesões coronárias em vasos de fino calibre, numa coorte de pacientes do mundo real submetidos a intervenção coronária percutânea (ICP) com stents farmacológicos. Método: Entre maio de 2002 e dezembro de 2009, 1.380 pacientes consecutivos do Registro DESIRE (Drug Eluting Stents In the Real World), com 1.683 lesões em vasos de fino calibre $(\leq 2,5 \mathrm{~mm}$ de diâmetro), foram submetidos a ICP, eletiva ou de urgência, com implante de 1.818 stents farmacológicos $\left(\right.$ Cypher $^{\mathrm{TM}}, 89 \%$; Taxus $^{\mathrm{TM}}$, 7,5\%; Xience $\mathrm{V}^{\mathrm{TM}} /$ Promus $^{\mathrm{TM}}$, 3\%; Endeavor ${ }^{\mathrm{TM}}, 0,33 \%$; Biomatrix $^{\mathrm{TM}}, 0,2 \%$ ) e incluídos neste estudo. O seguimento clínico de até 7 anos (mediana, 2,8 anos) foi completo em $98 \%$, sendo obtido com 1, 6 e 12 meses e, então, anualmente. Tivemos como objetivo determinar as taxas de eventos cardíacos adversos maiores (ECAM) ao longo do período do acompanhamento clínico. Resultados: A média de idade foi de $64,5 \pm 11,7$ anos, com predomínio de pacientes do sexo masculino $(76,7 \%)$. Diabetes melito foi encontrado em $31,6 \%$ dos casos e $9,8 \%$ eram portadores de insuficiência renal crônica. A artéria descendente anterior foi o vaso mais frequentemente tratado $(43,4 \%)$ e dois terços das lesões eram de alta complexidade (B2/C). Sucesso angiográfico do procedimento foi obtido em $98,8 \%$ dos casos. A taxa de ECAM foi de $12,8 \%$ durante o seguimento, com $4,5 \%$ de óbito cardíaco, 4,2\% de infarto agudo do miocárdio, 4,5\% de nova revascularização da lesão-alvo e 1,5\% de trombose do stent. Conclusão: No Registro DESIRE, o tratamento de vasos de pequeno calibre com stents farmacológicos, em pacientes não-selecionados, associou-se a excelentes resultados agudos e tardios, e a baixas taxas de trombose ao longo da evolução.

DESCRITORES: Stents farmacológicos. Angioplastia transluminal percutânea coronária. Reestenose coronária.

\section{ABSTRACT}

\section{Drug Eluting Stents for the Treatment of Small Vessels: Very Late Experience (Up to 7 Years) of the DESIRE Registry}

Background: In this study, we sought to evaluate the clinical outcomes of patients with coronary lesions in small vessels in a cohort of real world patients undergoing percutaneous coronary intervention ( $\mathrm{PCI}$ ) with drug-eluting stents (DES). Method: Between May 2002 and December 2009, 1,380 consecutive patients from the DESIRE Registry (Drug Eluting Stents In The Real World), with 1,683 lesions in small vessels $(<2.5 \mathrm{~mm}$ in diameter) were consecutively submitted to elective or emergency $\mathrm{PCl}$, with 1,818 DES (Cypher ${ }^{\mathrm{TM}}$, 89\%; Taxus $^{\mathrm{TM}}, 7.5 \%$; Xience $\mathrm{V}^{\mathrm{TM}} /$ Promus $^{\mathrm{TM}}$, 3\%; Endeavor, $0.33 \%$; Biomatrix ${ }^{\mathrm{TM}}, 0.2 \%$ ) and included in this study. The clinical follow-up of up to 7 years (median, 2.8 years) was completed for $98 \%$, and was obtained at 1, 6, 12 months and then annually. Our objective was to determine the rates of major cardiac events (MACE) during clinical follow-up. Results: Mean age was $64.5 \pm 11.7$ years, with a prevalence of male patients $(76.7 \%)$. Diabetes mellitus was observed in $31.6 \%$ of the cases and $9.8 \%$ had chronic renal failure. Left anterior descending artery was the most frequent vessel treated $(43.4 \%)$ and two-thirds of the lesions were complex lesions (B2/C). Angiographic success was obtained in 98.8\% of the cases. The rate of MACE was $12.8 \%$ during followup with $4.5 \%$ of cardiac death, $4.2 \%$ of acute myocardial infarction, $4.5 \%$ of repeat target lesion revascularization and $1.5 \%$ of stent thrombosis. Conclusion: In the DESIRE Registry, the use of DES to treat small vessels in non-selected patients was associated with excellent early and late outcomes and low thrombosis rates.

KEY-WORDS: Drug-eluting stents. Angioplasty, transluminal, percutaneous coronary. Coronary restenosis.

\footnotetext{
1 Instituto de Ensino e Pesquisa do Hospital do Coração (HCor) Associação do Sanatório Sírio - São Paulo, SP, Brasil.

Correspondência: J. Eduardo Sousa. Rua Desembargador Eliseu Guilherme, 147 - Paraíso - São Paulo, SP, Brasil - CEP 04004-030 E-mail: jesousa@uol.com.br

Recebido em: 7/8/2010 • Aceito em: 12/9/2010
} 
0 tratamento percutâneo da doença coronária em vasos de fino calibre representa um dos desafios da cardiologia intervencionista, em decorrência das elevadas taxas de reestenose após o tratamento com balão ou stents não-farmacológicos. Diversos estudos já demonstraram que quanto menor o vaso tratado maior o impacto da perda luminal tardia nas taxas de reestenose. ${ }^{1-3}$

O advento dos stents farmacológicos na prática clínica promoveu sensível melhora dos resultados no tratamento percutâneo da doença coronária, demonstrando ser mais eficazes em reduzir a proliferação neointimal, a reestenose angiográfica e a necessidade de revascularização da lesão-alvo em populações selecionadas de estudos randomizados. Dessa forma, a partir de 2002, quando os stents farmacológicos foram aprovados para uso clínico no Brasil, sua utilização vem se ampliando em cenários de maior complexidade tanto clínica como angiográfica.

Nesse contexto, o objetivo da presente análise foi identificar as taxas de eventos cardíacos adversos maiores em pacientes submetidos a intervenção coronária percutânea em vasos de fino calibre tratados exclusivamente com stents farmacológicos.

\section{MÉTODO}

\section{Casuística e desenho do estudo}

O Registro Drug Eluting Stents In the Real World (DESIRE) é um estudo clínico prospectivo, não-randomizado, de braço único, com inclusão consecutiva de pacientes (em andamento), iniciado em maio de 2002, realizado em uma única instituição (Hospital do Coração - Associação do Sanatório Sírio - São Paulo, $\mathrm{SP}$, Brasil), tendo como objetivo investigar a evolução clínica de pacientes tratados com stents farmacológicos. Os resultados gerais do DESIRE já foram previamente publicados, razão pela qual não nos deteremos em pormenores do registro. ${ }^{4,5}$

Na presente análise, avaliamos 1.380 pacientes com sintomas isquêmicos e/ou exames não-invasivos indicativos de isquemia, com lesão $\geq 50 \%$ em vasos com diâmetro $\leq 2,5 \mathrm{~mm}$ à angiografia (avaliação visual) e anatomia favorável para intervenção coronária percutânea. O estudo está em consonância com a Declaração de Helsinque no que se refere à investigação em humanos, e foi aprovado pelo Comitê de Ética da referida instituição.

\section{Procedimento}

O implante do stent farmacológico seguiu a técnica contemporânea estabelecida nas diretrizes atuais e a estratégia final do procedimento ficou a critério do operador.

Nos pacientes com síndrome coronária aguda, o estudo angiográfico e a intervenção coronária percutânea, quando indicada, foram realizados nas primei- ras 48 horas da hospitalização. No pré-tratamento foram administrados ácido acetilsalicílico e clopidogrel nas doses de 100-300 mg e 300-600 mg, respectivamente, 24 horas antes da intervenção, nos casos eletivos, 3 a 6 horas antes, nas síndromes coronárias agudas sem supradesnivelamento do segmento ST, e imediatamente antes, nos casos de intervenção coronária primária. Após o procedimento, o ácido acetilsalicílico era mantido indefinidamente, na dose de 100-200 mg/dia, e o clopidogrel, na dose de $75 \mathrm{mg} /$ dia, mantido por de 3 a 6 meses até 2006. Após essa data, seguindo as recomendações do Food and Drug Administration (FDA), o uso do clopidogrel foi estendido para 12 meses. Durante o procedimento, foi administrada heparina endovenosa (70-100 unidades $/ \mathrm{kg}$ ) para manter o tempo de coagulação ativada $>250$ segundos (ou > 200 segundos, no caso de ser administrado conjuntamente um inibidor de glicoproteína IIb/IIla). Durante a hospitalização, todos os pacientes foram submetidos a avaliação com eletrocardiograma e marcadores bioquímicos, incluindo creatina fosfoquinase (CPK) e creatina quinase fração $\mathrm{MB}$ massa (CK-MB), pré-procedimento (<24 horas), 18-24 horas pós-procedimento, e diariamente até a alta hospitalar.

Quando necessária, a pré-dilatação era realizada com balões curtos insuflados a baixas pressões, visando a evitar injúria nos segmentos adjacentes à lesão. $\mathrm{O}$ stent foi implantado de forma a recobrir por completo a lesão; quando necessário, mais de um stent foi empregado com a sobreposição de suas bordas. Após o implante do stent, a pós-dilatação, quando indicada, foi realizada com balões de extensão menor que a do stent implantado, não ultrapassando suas bordas. Os seguintes stents farmacológicos foram utilizados: Cypher ${ }^{\mathrm{TM}}$ (Cordis, Johnson \& Johnson, Warren, Estados Unidos), Taxus ${ }^{\mathrm{TM}}$ (Boston Scientific, Natick, Estados Unidos), Endeavor ${ }^{\mathrm{TM}}$ (Medtronic, Santa Rosa, Estados Unidos), Xience ${ }^{\mathrm{TM}}$ (Abbott Vascular, Abbott Park, Estados Unidos) e Biomatrix ${ }^{\mathrm{TM}}$ (Biosensor International, Cingapura). A escolha do tipo de stent farmacológico ficou a critério do operador.

A angiografia coronária quantitativa foi realizada imediatamente antes e após o implante do stent, por operadores experientes, utilizando um sistema de análise quantitativa com detecção semiautomática das bordas (Quantcor QCA-ACOM.PC versão 4.0 - Siemens, Munique, Alemanha). A análise do segmento tratado envolveu a área coberta pelo stent e as bordas $(5 \mathrm{~mm}$ ) proximal e distal. As mensurações angiográficas incluíram os diâmetros de referência proximal, distal e interpolado, o diâmetro mínimo da luz, o porcentual de obstrução, a extensão da lesão, e o ganho imediato (obtido pela diferença entre o diâmetro mínimo da luz imediatamente após e antes do procedimento).

\section{Objetivos do estudo, definições e seguimento clínico}

O objetivo primário deste estudo foi a avaliação da ocorrência de eventos cardíacos adversos maiores 
(morte cardíaca, infarto agudo do miocárdio, revascularização da lesão-alvo) e trombose do stent na fase hospitalar e no seguimento clínico a longo prazo.

Os óbitos foram classificados em cardíacos e nãocardíacos, sendo os óbitos de causas indeterminadas relatados como cardíacos. O diagnóstico de infarto agudo do miocárdio foi definido como aparecimento de nova onda $Q$ patológica em $\geq 2$ derivações contíguas do eletrocardiograma e/ou elevação da CK-MB massa $>3$ vezes o limite superior da normalidade. A revascularização da lesão-alvo foi definida como nova reintervenção, percutânea ou cirúrgica, resultante da recorrência de obstrução (> 50\%) no segmento tratado.

A trombose do stent foi definida de acordo com a classificação proposta pelo Academic Research Consortium (ARC): definitiva (síndrome coronária aguda com confirmação angiográfica ou anatomopatológica da oclusão do vaso), provável (ocorrência de morte súbita $\leq 30$ dias após o procedimento índice ou infarto agudo do miocárdio relacionado à região da artéria tratada, mesmo sem confirmação angiográfica) e possível (ocorrência de morte súbita > 30 dias após o procedimento índice). Ainda quanto à distribuição temporal, a trombose do stent foi classificada como aguda ( $\leq 24$ horas do procedimento), subaguda ( $>24$ horas e $\leq 30$ dias), tardia (>1 mês e $\leq 12$ meses) e muito tardia (> 12 meses pós-procedimento).

O sucesso angiográfico foi definido como a presença de lesão residual $<20 \%$, com fluxo coronário final TIMI 3 na ausência de trombos e/ou dissecções.

O sucesso do procedimento foi definido como sucesso angiográfico na ausência de eventos cardíacos maiores.

A insuficiência renal crônica foi definida como taxa de filtração glomerular (clearance de creatinina) $<60 \mathrm{ml} / \mathrm{min} / 1,73 \mathrm{~m}^{2}$.

A função ventricular esquerda foi avaliada pelo cálculo da fração de ejeção (\%FE) e classificada como: normal (\%FE $\geq 55 \%$ ), disfunção discreta (\%FE $\geq 40 \%$ e $<55 \%$ ), disfunção moderada ( $\%$ FE $\geq 30 \%<40 \%$ ) e disfunção grave $(\% \mathrm{FE}<30 \%)$.

O seguimento clínico foi realizado 1 mês, 6 e 12 meses após o procedimento e anualmente a seguir e obtido por visita médica ou por contato telefônico, seguindo protocolo pré-definido.

As variáveis categóricas estão descritas como porcentuais e as variáveis contínuas como média \pm desvio padrão. A sobrevida livre de eventos cardíacos maiores foi estimada pela curva de Kaplan-Meier. Utilizou-se o programa estatístico SPSS (versão 16.0) para realizar esta análise.

\section{RESULTADOS}

Entre maio de 2002 e dezembro de 2009, foram tratados, no total, 1.380 pacientes portadores de le- sões coronárias em vasos de fino calibre, perfazendo 41,5\% da população total do Registro DESIRE.

A Tabela 1 apresenta as principais características dos pacientes incluídos neste estudo. A maioria era do sexo masculino $(76,7 \%)$, com média de idade de $64,5 \pm 11,7$ anos. Cerca de um terço dos pacientes $(31,6 \%)$ era portador de diabetes melito, $25,4 \%$ tinham história de intervenção coronária percutânea prévia e $27,2 \%$ tinham cirurgia de revascularização miocárdica prévia. Síndrome coronária aguda como apresentação clínica inicial ocorreu em 40,2\% dos casos $(25,8 \%$ de angina instável e $14,4 \%$ de infarto agudo do miocárdio). A artéria descendente anterior foi o território mais frequentemente tratado $(43,4 \%)$.

A angiografia quantitativa pré-intervenção demonstrou que o diâmetro de referência e a extensão das lesões tratadas eram de 2,33 \pm 0,18 mm e 16,6 9 mm,

\section{TABELA 1}

Características clínicas e angiográficas basais

\begin{tabular}{lc}
\hline & $\mathbf{1 . 3 8 0}$ pacientes \\
Variável & $\mathbf{( 1 . 6 8 3}$ lesões) \\
\hline Média de idade, anos & $64,5 \pm 11,7$ \\
Sexo feminino, \% & 23,3 \\
Diabetes melito, \% & 31,6 \\
HAS, \% & 77,9 \\
Dislipidemia, \% & 62,8 \\
Tabagismo atual, \% & 8,9 \\
História familiar de DAC, \% & 37,3 \\
IAM prévio, \% & 23,9 \\
Intervenção coronária & 25,4 \\
percutânea prévia, \% & 27,2 \\
CRM prévia, \% & 2,9 \\
AVC prévio, \% & 3,9 \\
Doença vascular periférica, \% & 9,8 \\
Insuficiência renal, \% & \\
Apresentação clínica, \% & 24,2 \\
Angina estável & 25,8 \\
Angina instável & 14,4 \\
IAM & 35,4 \\
Isquemia silenciosa & \\
Território tratado, \% & \\
Artéria descendente anterior & 33,4 \\
Artéria circunflexa & 35,5 \\
Artéria coronária direita & 20,9 \\
Doença multiarterial, \% & 14,4 \\
Disfunção de VE (FE < 40\%), \% & \\
\hline AVC = acidente vascular cerebral; CRM cirurgia de \\
revascularização miocárdica; DAC doença arterial co- \\
ronária; FE = fração de ejeção; HAS = hipertensão arterial \\
sistêmica; IAM = infarto agudo do miocárdio; VE = ven- \\
trículo esquerdo. \\
\hline
\end{tabular}


respectivamente. Após a intervenção, a lesão residual foi de $4,2 \pm 4 \%$. O stent Cypher ${ }^{\mathrm{TM}}$ foi utilizado na maioria dos casos (89\%). As taxas de fluxo coronário TIMI 3 e de sucesso angiográfico foram de 99,6\% e $98,8 \%$, respectivamente (Tabela 2 ).

O seguimento clínico em até 7 anos (mediana, $2,8 \pm 1,8$ anos) foi obtido em $98,1 \%$ dos pacientes, não sendo o reestudo angiográfico obrigatório pelo protocolo. Durante o período de seguimento, a taxa combinada de eventos cardíacos adversos maiores foi de 12,8\% (Figura 1), incluindo óbito cardíaco em 4,5\%, infarto agudo do miocárdio em $4,2 \%$ e revascularização da lesão-alvo em 4,5\% dos pacientes.

A taxa de trombose de stent nessa população foi de $1,5 \%$ (21 casos), sendo 14 casos (66\%) classificados como trombose definitiva. Quanto à distribuição temporal, 9 tromboses definitivas ocorreram no primeiro ano do procedimento (6 subagudas e 3 tardias), e 5 ocorreram após o primeiro ano (muito tardias).

TABELA 2

Análise angiográfica quantitativa e características do procedimento

\begin{tabular}{|c|c|}
\hline Características & $\begin{array}{c}1.683 \text { lesões/ } \\
1.818 \text { stents }\end{array}$ \\
\hline Extensão da lesão, mm & $16,6 \pm 9$ \\
\hline Extensão do stent, mm & $19,2 \pm 6,2$ \\
\hline Extensão do stent/extensão da lesão, mm & 1,2 \\
\hline \multicolumn{2}{|l|}{ Stent implantado, \% } \\
\hline Cypher ${ }^{\mathrm{TM}}$ & 89 \\
\hline Taxus $^{\text {TM }}$ & 7,5 \\
\hline Xience $^{T M}$ & 3 \\
\hline Endeavor $^{\mathrm{TM}}$ & 0,3 \\
\hline Biomatrix $^{\top M}$ & 0,2 \\
\hline Pré-dilatação, \% & 64,1 \\
\hline Pós-dilatação, \% & 50 \\
\hline Pressão máxima de liberação, atm & $13,3 \pm 2,1$ \\
\hline Stents por lesão/por paciente & $1,1 / 1,3$ \\
\hline Diâmetro de referência do vaso, mm & $2,33 \pm 0,18$ \\
\hline Diâmetro dos stents, mm & $2,52 \pm 0,19$ \\
\hline \multicolumn{2}{|l|}{ Diâmetro luminal mínimo, mm } \\
\hline Pré-procedimento & $0,76 \pm 0,73$ \\
\hline Pós-procedimento & $2,36 \pm 0,74$ \\
\hline \multicolumn{2}{|l|}{ Diâmetro de estenose, \% } \\
\hline Pré-procedimento & $68,1 \pm 10,7$ \\
\hline Pós-procedimento & $4,2 \pm 4$ \\
\hline Ganho agudo, mm & $1,61 \pm 0,43$ \\
\hline Fluxo TIMI 3, \% & 99,6 \\
\hline Sucesso angiográfico, \% & 98,8 \\
\hline Sucesso do procedimento, $\%$ & 98 \\
\hline
\end{tabular}

\section{DISCUSSÃO}

O principal achado do presente estudo refere-se à segurança e à eficácia bastante tardia dos stents farmacológicos no tratamento de vasos finos, cenário em que historicamente a intervenção percutânea com cateter-balão ou stents não-farmacológicos apresenta resultados menos expressivos.

O conceito de vaso fino varia bastante entre diversos estudos publicados, o que dificulta a tarefa de pôr em contexto nossos resultados diante de outros estudos. Enquanto alguns autores consideram vasos finos aqueles com calibre $\leq 3 \mathrm{~mm}$, outros utilizam como valor de referência diâmetros de 2,75 mm e 2,5 mm. O que se sabe, de fato, é que há progressivo aumento das taxas de eventos adversos, sobretudo reestenose, proporcionalmente à diminuição do diâmetro de referência do vaso tratado. Na presente análise foram incluídos os vasos que consideramos realmente finos, ou seja, aqueles com diâmetro $\leq 2,5 \mathrm{~mm}$.

Ainda que os stents não-farmacológicos tenham impulsionado de forma marcante o desenvolvimento da cardiologia intervencionista, melhorando sobremaneira os resultados imediatos e tardios das intervenções apenas com cateter-balão, o uso desses dispositivos em alguns cenários de maior complexidade clínica e anatômica, dentre os quais tratamento de vasos finos, ainda representa um desafio, dada as elevadas taxas de eventos adversos, sobretudo recorrência da lesão na lesão-alvo. Recente meta-análise a respeito do uso de stents não-farmacológicos no tratamento de lesões em vasos finos demonstrou, ao final de um ano, taxa de eventos cardíacos adversos maiores de $27,8 \%$, sobretudo em decorrência da elevada necessidade de nova revascularização da lesão-alvo $(17,6 \%){ }^{5}$

Com o advento dos stents farmacológicos, com sua marcante capacidade de reduzir a proliferação neointimal e, consequentemente, a reestenose, nos mais

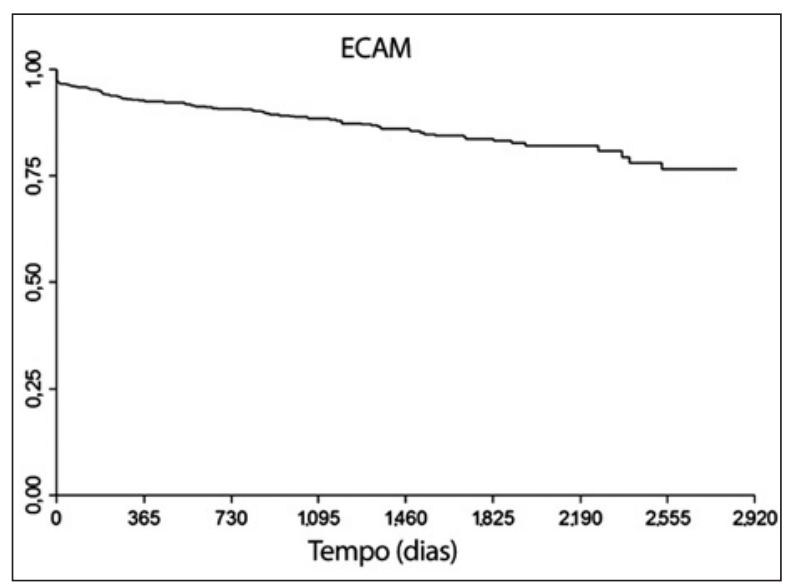

Figura 1 - Curva de sobrevivência livre de eventos cardíacos adversos maiores (ECAM). 
variados e complexos cenários clínicos e angiográficos, o tratamento de lesões em vasos finos ganhou novo alento.

No estudo Canadian study of the sirolimus-eluting stent in the treatment of patients with long de novo lesions in small native coronary arteries (C-SIRIUS), Schampaert et al. ${ }^{6}$ avaliaram de forma randomizada o desempenho do stent farmacológico Cypher ${ }^{\mathrm{TM}}$ vs. seu análogo não-farmacológico no tratamento de 100 pacientes com lesões longas em vasos finos. Nesse estudo, o uso do stent farmacológico associou-se a marcante redução da taxa de reestenose ao final de nove meses ( $4 \%$ vs. $18 \% ; \mathrm{P}=0,05){ }^{6}$

Em 2007, Seabra-Gomes et al. ${ }^{7}$ publicaram os resultados do estudo multicêntrico PORTO, que avaliou o desempenho do stent farmacológico Cypher ${ }^{\mathrm{TM}}$ no tratamento de 324 lesões situadas em vasos de diâmetro $\leq 2,5 \mathrm{~mm}$. No estudo angiográfico de seis meses, a perda luminal no interior do stent foi de $0,07 \pm 0,37 \mathrm{~mm}$, a reestenose binária foi de 9,1\%, a taxa de eventos cardíacos adversos maiores ao final de um ano foi de $8,6 \%$, com apenas $5,6 \%$ de novas intervenções na lesão-alvo. ${ }^{7}$

A série de estudos TAXUS avaliou o desempenho do stent paclitaxel no tratamento de vasos finos. No estudo TAXUS IV, 176 pacientes com lesões em vasos de $2,5 \mathrm{~mm}$ foram randomizados para receber o stent Taxus $^{\text {TM }}$ ou seu análogo sem fármaco. ${ }^{8}$ Ao final de 12 meses, os pacientes que receberam stent farmacológico tiveram marcante redução da necessidade de nova intervenção na lesão-alvo $(5,6 \%$ vs. $20,6 \%$; $P<0,001) .{ }^{8}$ No estudo TAXUS $V$, analisou-se um subgrupo de pacientes $(17,6 \%$ do total da população do estudo) randomizados para receber stent Taxus ${ }^{\mathrm{TM}} 2,25 \mathrm{~mm}$ ou seu equivalente não-farmacológico. Mais uma vez, o uso de stent farmacológico em vasos de muito pequeno calibre associou-se a significante redução das taxas de reestenose $(31,2 \%$ vs. $49,4 \% ; P=0,03) .{ }^{9}$

Na comparação direta entre os stents farmacológicos com sirulimus e paclitaxel para tratamento de pequenos vasos, o uso de stent com sirulimus esteve associado a menores taxas de perda luminal intrastent, reestenose binária e necessidade de nova revascularização da lesão-alvo. ${ }^{10}$

Os resultados do presente estudo corroboram a eficácia dos stents farmacológicos nesse cenário de maior complexidade angiográfica. A despeito de a população aqui avaliada ter um perfil clínico e angiográfico mais complexo que os pacientes incluídos nos estudos controlados supracitados, as taxas de eventos cardíacos adversos maiores e de reestenose mimetizaram os resultados dos ensaios randomizados. Esses achados devem-se, em parte, a dois principais fatores: uso preponderante de stents com sirolimus e ausência de reestudo angiográfico de rotina. Aliado a isso, o uso rotineiro de angiografia quantitativa on-line para guiar a intervenção e a realização de pré e pós-dilatação sempre que necessário ajudam a explicar os excelentes resultados aqui apresentados.

\section{Limitações do estudo}

Uma das principais limitações deste estudo referese à ausência de um grupo controle tratado com stents não-farmacológicos, uma vez que o Registro DESIRE representa a experiência clínica de uma única instituição, onde os stents farmacológicos vêm sendo utilizados como estratégia preferencial em pacientes com indicação de intervenção coronária percutânea no mundo real. Ademais, a predominância na utilização de um stent farmacológico (Cypher ${ }^{\mathrm{TM}}$ ) sobre os demais em proporções não-balanceadas, com indicações baseadas na disponibilidade, nas características anatômicas e da lesão, na apresentação clínica e na preferência do operador, impossibilitaram a comparação de diferentes stents farmacológicos disponíveis nesse cenário.

\section{CONCLUSÕES}

Na presente análise do Registro DESIRE, a utilização dos stents farmacológicos associou-se a excelentes resultados agudos e tardios no tratamento de lesões de vasos de fino calibre em pacientes não selecionados. Merece destaque o fato de no seguimento clínico tardio não terem sido observados problemas relacionados à segurança desses dispositivos, com baixas taxas de trombose em todas as fases de evolução desses pacientes.

\section{CONFLITO DE INTERESSES}

Os autores declararam inexistência de conflito de interesses relacionado a este manuscrito.

\section{REFERÊNCIAS}

1. Lee CW, Park DW, Lee BK, Kim YH, Hong MK, Kim JJ, et al. Predictors of restenosis after placement of drug-eluting stents in one or more coronary arteries. Am J Cardiol. 2006; 97(4):506-11.

2. Dussaillant GR, Mintz GS, Pichard AD, Kent KM, Satler LF, Popma J, et al. Small stent size and intimal hyperplasia contribute to restenosis: a volumetric intravascular ultrasound analysis. J Am Coll Cardiol. 1995;26(3):720-4.

3. Mauri L, Orav EJ, O'Malley AJ, Moses JW, Leon MB, Holmes $\mathrm{DR}$, et al. Relationship of late loss in lumen diameter to coronary restenosis in sirolimus-eluting stents. Circulation. 2005; 111(3):321-7.

4. Sousa A, Costa JR Jr, Moreira AC, Cano M, Maldonado G, Costa RA, et al. Drug-Eluting Stents in the Real World (DESIRE) Registry. Long-term clinical outcomes of the DrugEluting Stents in the Real World (DESIRE) Registry. J Interv Cardiol. 2008;21(4):307-14.

5. Agostoni P, Biondi-Zoccai GG, Gasparini GL, Anselmi M, Morando G, Turri M, et al. Is bare-metal stenting superior to balloon angioplasty for small vessel coronary artery disease? Evidence from a meta-analysis of randomized trials. Eur Heart J. $2005 ; 26(9): 881-9$.

6. Schampaert E, Cohen EA, Schluter M, Reeves F, Traboulsi M, 
Nogueira EF, et al. Stents Farmacológicos para o Tratamento de Coronárias de Fino Calibre: Experiência Muito Tardia (Até 7 Anos) do Registro DESIRE. Rev Bras Cardiol Invasiva. 2010;18(3):288-93.

Title LM, et al. for the C-SIRIUS Investigators. The Canadian study of the sirolimus-eluting stent in the treatment of patients with long de novo lesions in small native coronary arteries (C-SIRIUS). J Am Coll Cardiol. 2004;43(6):1110-5.

7. Seabra-Gomes R, Sousa JE, Sousa A, Teles R, Pereira H, Farto Abreu P, et al. Small coronary arteries treated with sirolimuseluting stents: one-year results of the PORTO multicentre registry. Eurolntervention. 2007;3(2):197-205.

8. Stone GW, Ellis SG, Cox DA, Hermiller J, O'Shaughnessy C, Mann JT, et al. One-year clinical results with the slow- release, polymer-based, paclitaxel-eluting TAXUS stent: the TAXUS-IV trial. Circulation. 2004;109(16):1942-7.

9. Stone GW, Ellis SG, Cannon L, Mann JT, Greenberg JD, Spriggs D, et al. Comparison of a polymer based paclitaxeleluting stent with a bare metal stent in patients with complex coronary artery disease: a randomized controlled trial. JAMA. 2005;294(10):1215-23.

10. Mehilli J, Dibra A, Kastrati A, Pache J, Dirschinger J, Schömig $A$, et al. Randomized trial of paclitaxel and sirolimus-eluting stents in small coronary vessels. Eur Heart J. 2006;27(3):260-6. 